\title{
Solving Finite Length Beam Equation by the Haar Wavelet Method
}

\author{
Dr.G.Hariharan \\ Department of Mathematics \\ SASTRA University \\ Thanjavur-613 401 \\ Tamilnadu, India
}

\begin{abstract}
The beam is assumed partitioned into several finite elements and the deflection of the beam is required to be a positive quantity along the whole beam so that the related fundamental fourthorder ordinary differential equation can continuously holds good. In this paper, we apply Haar wavelet methods to solve finite-length beam differential equations with initial or boundary conditions known. An operational matrix of integration based on the Haar wavelet is established and the procedure for applying the matrix to solve the differential equations is formulated. The fundamental idea of Haar wavelet method is to convert the differential equations into a group of algebraic equations, which involves a finite number of variables. Illustrative example is given to confirm the efficiency and the accuracy of the proposed algorithm. The results show that the proposed way is quite reasonable when compared to exact solution.
\end{abstract}

\section{Keywords}

Haar wavelets; Ordinary differential equation;finite-length beam; computationally attractive

\section{INTRODUCTION}

The important problem of the bending of a beam on an elastic foundation has been the subject of a number of investigations. One of the earliest of these investigations was the work of Winkler [18] who assumed that, for the purposes of analysis, the elastic foundation could be replaced by a continuous set of springs, each of which can be deflected independently of the others.

Elastic beams under bending conditions resting on an elastic foundation have been of great importance in applied and computational mechanics long ago mainly because of their practical applications in engineering, especially in civil engineering. The related fundamental treatise is still that by Hetrnyi [8], where the classical Winkler hypotheses about the elastic foundation were assumed valid. The related famous fourth-order ordinary differential equation has the well-known form [8].

$$
E_{b} I \frac{d^{4} y}{d x^{4}}+k_{0} b y=b P x
$$

\footnotetext{
where $E_{b} I$ is the elasticity modules and section moment of inertia for the material of the beam; $b$ is the width of the beam; $k_{0}$

0 is the centralized bending coefficient for the unit length of
}

the beam; $P x$ is the external load; $y$ is the flexibility of the beam. Dynamic response of elastic-plastic continuous beams under short pulse loading was considered by Lepik $[11,12,13]$. The problem of an infinite length beam bonded to elastic halfspace has been investigated by Blot [2]. A different method and different assumptions are used herein for a finite length beam. AlvarezDíaz et. al [1] had solved beam and finite elements by using Daubechies wavelets. Pavlovid and Tsikkos [17] had discussed the Beams on quasi-Winkler foundations. Xia and Zhang [19] had established a numerical method for critical buckling load for a beam supported elastic foundation.

Haar wavelets have been applied extensively for signal processing in communications and physics research, and more mathematically focused on differential equations and even nonlinear problems. After discretizing the differential equation in a convenient way like the finite difference approximation, wavelets can be used for algebraic manipulations in the system of equations obtained which may lead to better condition number of the resulting system.

The previous work in system analysis via Haar wavelets was led by Chen and Hsiao [4], who first derived a Haar operational matrix for the integrals of the Haar function vector and put the application for the Haar analysis into the dynamical systems. Then, the pioneer work in state analysis of linear time delayed systems via Haar wavelets was laid down by Hsiao [10], who first proposed a Haar product matrix and a coefficient matrix. In order to take the advantages of the local property, many authors researched the Haar wavelet to solve differential and integral equations.

In this paper, our work stems mainly from the Haar wavelet method and we established a clear procedure for solving the differential equations via Haar wavelet. The Haar wavelet method, which will exhibit several advantageous features:

i) Very high accuracy fast transformation and possibility of implementation of fast algorithms compared with other known methods.

ii) The simplicity and small computation costs, resulting from the sparsity of the transform matrices and the small number of significant wavelet coefficients.

iii)The method is also very convenient for solving the boundary value problems, since the boundary conditions are taken care of automatically.

Haar wavelets (which are Daubechies of order 1) consists of piecewise constant functions and are therefore the simplest orthonormal wavelets with a compact support. The main advantage of the Haar wavelet is that simplicity gets to some extent lost [3]. Lepik $[14,15,16]$ had solved higher order as well as nonlinear ODEs by using Haar wavelet method. Dai and Cochran [5] had introduced wavelet collocation method for 
optimal Control Problems. Hariharan et.al [7] had established the Haar wavelet method for depth profile of soil temperature. Hsiao and Wang [9] had solved nonlinear stiff systems by Haar wavelet method.

We organized our paper as follows. In section 2, the Haar wavelet is introduced and an operational matrix is established. In section 3, we established operational matrix and use Haar wavelets to solve differential equation. Because of the local property of the powerful Haar wavelet, the new method is simpler in reasoning as well as in calculation. In section 4 , we presented the product operational matrix of the Haar wavelet. In section 5, we established the Procedure of Haar wavelet method for ODE. In section 6, we implemented the Haar solution for the finite-length beam equation. Concluding remarks are given in Section 7.

\section{SOME PROPERTIES OF HAAR WAVELETS}

Haar wavelet is the simplest wavelet. Haar transform or Haar wavelet transform has been used as an earliest example for orthonormal wavelet transform with compact support. The Haar wavelet transform is the first known wavelet and was proposed in 1910 by Alfred Haar [6]. They are step functions (piecewise constant functions) on the real line that can take only three values. Haar wavelets, like the well-known Walsh functions (Rao 1983), form an orthogonal and complete set of functions representing discretized functions and piecewise constant functions. A function is said to be piecewise constant if it is locally constant in connected regions.

The Haar transform is one of the earliest examples of what is known now as a compact, dyadic, orthonormal wavelet transform. The Haar function, being an odd rectangular pulse pair, is the simplest and oldest orthonormal wavelet with compact support. In the mean time, several definitions of the Haar functions and various generalizations have been published and used. They were intended to adopt this concept to some practical applications as well as to extend its applications to different classes of signals. Haar functions appear very attractive in many applications as for example, image coding, edge extraction, and binary logic design.

After discretizing the differential equations in a conventional way like the finite difference approximation, wavelets can be used for algebraic manipulations in the system of equations obtained which lead to better condition number of the resulting system.

For applications of the Haar transform in logic design, efficient ways of calculating the Haar spectrum from reduced forms of Boolean functions are needed.

The Haar wavelet family for $t \in[0,1]$ is defined as follows.

$$
h_{i}(t)=\left\{\begin{array}{l}
1, \quad \text { for } t \in\left[\frac{k}{m}, \frac{k+0.5}{m}\right) \\
-1, \text { for } t \in\left[\frac{k+0.5}{m}, \frac{k+1}{m}\right)(2) \\
0, \quad \text { elsewhere }
\end{array}\right.
$$

Integer $m=2^{j} \quad(j=0,1,2 \ldots J)$ indicates the level of the wavelet; $k=0,1,2, \ldots . . m-1$ is the translation parameter. Maximal level of resolution is J. The index $i$ is calculated according the formula $i=m+k+1$; in the case of minimal values $m=1, k=0$ we have $i=2$, the maximal value of $i$ is $i=2 M=2^{J+1}$. It is assumed that the value $i=1$ corresponds to the scaling function for which $h_{1} \equiv 1$ in 0,1 . Let us define the collocation points $t_{l}=(l-0.5) / 2 M,(l=1,2 \ldots .2 M)$ and discretise the Haar function $h_{i}(t)$; in this way we get the coefficient matrix $H(i, l)=\left(h_{i}\left(t_{l}\right)\right)$, which has the dimension $2 M \times 2 M$

In the wavelet analysis for a dynamic system, all functions need to be transformed into Haar series. Since the differentiation of Haar wavelets always results in impulse functions, which should be avoided, the integration of Haar wavelets is preferred, which should be expandable into Haar series with Haar coefficient matrix $P_{\text {[4] }}$

It is assumed that the value $i=1_{\text {corresponds to the scaling }}$ function for which

$$
h_{i} x=\left\{\begin{array}{l}
1 \text { for } x \in-1,1, \\
0 \text { elsewhere }
\end{array}\right.
$$

The operational matrix of integration $P$, which is a $2 \mathrm{M}$ square matrix, is defined by the equation

$$
P_{i}(x)=\int_{0}^{x} h_{i}(x) d x, \quad x \in 0,1
$$

In section 4 the second order differential equation was replaced with the system of first order equations. Here another variant of solution is demonstrated, by which the second order equation is directly solved. For this purpose the integral operator $Q$

is introduced.

$Q_{i} x=\int_{0}^{x} P_{i} x d x$

This integral can be evaluated according to (6). For instance for $2 M=4$ we have

$$
Q H=\frac{1}{128}\left[\begin{array}{cccc}
1 & 9 & 25 & 49 \\
1 & 9 & 23 & 31 \\
1 & 7 & 8 & 8 \\
0 & 0 & 1 & 7
\end{array}\right]
$$


The elements of the matrices $H, P$ and $Q$ can be evaluated according to (2), (3) and (4).

$$
\begin{aligned}
& H_{2}=\left(\begin{array}{cc}
1 & 1 \\
1 & -1
\end{array}\right), \quad P_{2}=\frac{1}{4}\left(\begin{array}{cc}
2 & -1 \\
1 & 0
\end{array}\right) \\
& H_{4}=\left[\begin{array}{cccc}
1 & 1 & 1 & 1 \\
1 & 1 & -1 & -1 \\
1 & -1 & 0 & 0 \\
0 & 0 & 1 & -1
\end{array}\right] \text {, } \\
& P_{4}=\frac{1}{16}\left[\begin{array}{cccc}
8 & -4 & -2 & -2 \\
4 & 0 & -2 & 2 \\
1 & 1 & 0 & 0 \\
1 & -1 & 0 & 0
\end{array}\right] \\
& P_{8}=\frac{1}{64}\left[\begin{array}{cccccccc}
32 & -16 & -8 & -8 & -4 & -4 & -4 & -4 \\
16 & 0 & -8 & 8 & -4 & -4 & 4 & 4 \\
4 & 4 & 0 & 0 & -4 & 4 & 0 & 0 \\
4 & 4 & 0 & 0 & -4 & 4 & 0 & 0 \\
1 & 1 & 2 & 0 & 0 & 0 & 0 & 0 \\
1 & 1 & -2 & 0 & 0 & 0 & 0 & 0 \\
1 & -1 & 0 & 2 & 0 & 0 & 0 & 0 \\
1 & -1 & 0 & -2 & 0 & 0 & 0 & 0
\end{array}\right]
\end{aligned}
$$

Chen and Hsiao [4] derived that the following matrix equation for calculating the matrix $P$ of order $m$ holds

$$
P_{m}=\frac{1}{2 m}\left(\begin{array}{cc}
2 m P_{m / 2} & -H_{m / 2} \\
H_{m / 2}^{-1} & O
\end{array}\right)
$$

where $O$ is a null matrix of order

$\frac{m}{2} \times \frac{m}{2}$

$H_{m \times m} \triangleq h_{m}\left(x_{0}\right), h_{m}\left(x_{1}\right), \ldots, h_{m}\left(x_{m-1}\right)$

$\frac{i}{m} \leq t<\frac{i+1}{m}$

and $H_{m \times m}^{-1}=\frac{1}{m} H_{m \times m}^{T} \operatorname{diag}(r)$

Here a diagonal matrix $\operatorname{diag} r$ is defined as

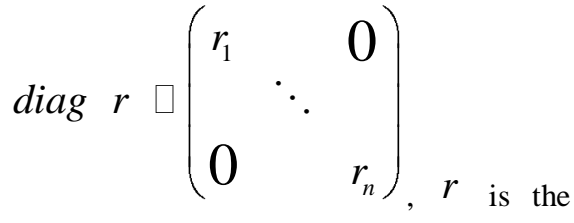

row vector (or column vector) with elements $r_{1}, r_{2}, \ldots r_{n}$.

It should be noted that calculations for $P_{m}$ and $H_{m}$ must be carried out only once; after that they will be applicable for solving whatever differential equations.

Table 1 Comparison of algorithmic complexity of the of the proposed method with FFT and WT

\begin{tabular}{llll}
\hline Series & $\begin{array}{l}\text { Numbers } \\
\text { additions }\end{array}$ & of & $\begin{array}{l}\text { Numbers } \\
\text { multiplications }\end{array}$ \\
\hline $\begin{array}{l}\text { Haar Transform } \\
\text { (HT) }\end{array}$ & $2 m-2$ & $m$ \\
$\begin{array}{l}\text { Walsh } \\
\text { Transform (WT) }\end{array}$ & $m \log _{2} m$ & $m$ \\
$\begin{array}{l}\text { Fast Fourier } \\
\text { Transform } \\
\text { (FFT) }\end{array}$ & $m \log _{2} m$ & $m \log _{2} m+1$ \\
\hline
\end{tabular}

The fast capability of HT should be impressive. Since $H$ and $H^{-1}$ contain many zeros, this phenomenon makes the Haar transform faster than the Fourier transform, and it is even faster than the Walsh transform. This is one of the reasons for rapid convergence of the Haar wavelet series. The number of additions and multiplications for these three transforms are shown in Table 1.

In practical applications, a small number of terms increases the calculation speed and saves memory storage; a large number of terms improve resolution accuracy. Therefore, a trade-off between calculation speed, memory saving, and the resolution accuracy must be considered in the analysis.

\section{FUNCTION APPROXIMATION}

Any square integrable function $y(x)$ in the interval 0,1 can be expanded by a Haar series of infinite terms

$$
y(x)=\sum_{i=0}^{\infty} c_{i} h_{i}(x), i \in 0 \cup N,
$$

where the Haar coefficients $c_{i}$ are determined as,

$$
\begin{gathered}
c_{0}=\int_{0}^{1} y(x) h_{0} x d x, \\
c_{n}=2^{j} \int_{0}^{1} y(x) h_{i}(x) d x \\
i=2^{j}+k, j \geq 0,0 \leq k<2^{j}, x \in 0,1
\end{gathered}
$$


such that the following integral square error $E$ is minimized:

$$
\begin{aligned}
& \varepsilon=\int_{0}^{1}\left[\begin{array}{lll}
y & x-\sum_{i=0}^{m-1} c_{i} h_{i} x
\end{array}\right]^{2} d x, \\
& m=2^{j}, \quad j \in 0 \cup N .
\end{aligned}
$$

Usually, the series expansion of (7) contains infinite terms for smooth $y(x)$. If $y(x)$ is piecewise constant by itself, or may be approximated as piecewise constant during each subinterval, then $y(x)$ will be terminated at finite $m$ terms, that is

$$
y(x)=\sum_{i=0}^{m-1} c_{i} h_{i}(x)=c_{(m)}^{T} h_{m}(x)
$$

where the coefficients $c_{m}^{T}$ and the Haar function vector $h_{m}(x)$ are defined as

$$
\begin{gathered}
c_{(m)}^{T}=\left[c_{0}, c_{1}, \ldots, c_{m-1}\right] \\
\text { and } \quad h_{m}(x)=\left[h_{0}(x), h_{1}(x), \ldots . ., h_{m-1}(x)\right]^{T}
\end{gathered}
$$

where ' $\mathrm{T}$ ' means transpose and $m=2^{j}$.

\section{HAAR WAVELET METHOD FOR SOLVING ODES}

For solving linear ordinary differential equation with $n^{\text {th }}$ order, say

$$
\begin{gathered}
a_{1} y^{n} \quad x+a_{2} y^{n-1} x+\ldots+a_{n} y x=f x, \\
\text { where } x \in A, B \text { and initial conditions }
\end{gathered}
$$

$$
y^{n-1} a, y^{n-2} a \ldots, y a \text { are known. }
$$

We follow the work done by Lepik [14]. Say we intend to do until $j$ level of resolution, hence we let $m=22^{j}$. The interval $A, B$ will be divided into $m$ subintervals, hence $\Delta x=\frac{B-A}{m}$ and the matrices are in the dimension of $m \times m$. Here we suggest the step-by-step procedures for easy understanding. Mainly, there are 5 steps in the procedure as follow.

Step 1: Let $y^{n} x=\sum_{i=1}^{m} a_{i} h_{i} x$, where $h$ is Haar matrix and $a_{i}$ is the wavelet coefficients.

Step 2: Obtain appropriate $v$ order of $y \quad x$ by using

$$
\begin{aligned}
& y^{v} x=\sum_{i=1}^{m} a_{i} P_{n-v, i} x \\
& +\sum_{\sigma=0}^{n-v-1} \frac{1}{\sigma !} x-A^{\sigma} y_{0}{ }^{v+\sigma}
\end{aligned}
$$

Step 3: Replace $y^{n} x$ and all the value of $y^{v} \quad x$ into the problem.

Step 4: Calculate the wavelet coefficients, $a_{i}$.

Step 5: Obtain the numerical solution for $y x$.

Fig.1 Algorithm for solving ordinary differential equation by Haar wavelet method

Step 2 is the key procedure where matrix $P_{n-v, i} x$ will be counted.

\section{FINITE-LENGTH BEAM EQUATION USING HAAR WAVELETS}

By the medium of Winkler, the basic differential equation for the flexibility of the beam is

$$
E_{b} I \frac{d^{4} y}{d x^{4}}+k_{0} b y=b P \quad x
$$

where $E_{b} I$ is the elasticity modules and section moment of inertia for the material of the beam; $b$ is the width of the beam; $k_{0}$ is the centralized bending coefficient for the unit length of the beam; $P x$ is the external load; $y$ is the flexibility of the beam.

The boundary conditions of finite-length beam cannot only be divided into the free end. Simple-supported and fixed end, but also be divided into any two terms among the three kinds conditions.

Table.1 All kinds of the conditions

\begin{tabular}{lll}
\hline $\begin{array}{l}\text { Fixed } \\
\text { End }\end{array}$ & $x=0$ or 1 & $y=y^{\prime}=0$ \\
\hline $\begin{array}{l}\text { Simple- } \\
\text { supported }\end{array}$ & $x=0$ or 1 & $y=y^{2}=0$ \\
Free end & $x=0$ or 1 & $y^{2}=y^{3}=0$ \\
\hline
\end{tabular}

For clarity in presentation, in the illustrative example, we assume that $E_{b} I=1, b=1, k_{0}=4, P \quad x=\cos (2 x)$.

differential equation for the flexibility of the beam is 
(11)

$$
y^{4} x+4 y x=\cos 2 x
$$

For solving this problem by the Haar wavelet method, we assume that $y^{4} x$ can be expanded in terms of Haar wavelets as formula (2). That is

$$
y^{4} x=\sum_{n=0}^{m-1} c_{n} h_{n} \quad x=c_{m}^{T} h_{m} x
$$

The function $\cos 2 x$ can be expanded into Haar 0,1

$$
\cos 2 x=\sum_{n=0}^{m-1} d_{n} h_{n} x=d_{m}^{T} h_{m} x
$$

where the vector $d_{m}^{T}$ can be obtained using formula (13).

Integrating formula (12) from 0 to $x$ and using formula (2), the variables $y^{3} x, y^{2} x, y^{\prime} x$ and y $x$

can be expressed as

$$
\begin{gathered}
y^{3} x=\int_{0}^{x} y^{4} t d t+y^{3} 0 \\
=\left[c_{m}^{T} P_{m}+f^{T} y^{3} \quad 0\right] h_{m} x \\
y^{2} x=\int_{0}^{x} y^{3} t d t+y^{2} 0 \\
=\left[\begin{array}{cccc}
c_{m}^{T} P_{m}^{2}+f^{T} y^{3} & 0 & P_{m} f^{T} y^{2} & 0
\end{array}\right] \\
h_{m} x
\end{gathered}
$$

$$
\begin{aligned}
& y^{\prime} x=\int_{0}^{x} y^{2} t d t+y^{\prime} 0 \\
& =\left[\begin{array}{l}
c_{m}^{T} P_{m}^{3}+f^{T} y^{3} \quad 0 \quad P_{m}^{2} f^{T} y^{2} \quad 0 \quad P_{m} \\
+f^{T} y^{\prime} 0
\end{array}\right.
\end{aligned}
$$$$
h_{m} x
$$

$$
y \quad x=\int_{0}^{x} y^{\prime} t d t+y \quad 0
$$

$$
=\left[\begin{array}{l}
c_{m}^{T} P_{m}^{4}+f^{T} y^{3} \quad 0 \quad P_{m}^{3} f^{T} y^{2} \quad 0 \quad P_{m}^{2} \\
+f^{T} y^{\prime} 0 P_{m} f^{T} y \quad 0
\end{array}\right]
$$

$h_{m} x$

where the vector $f$ is defined as

$$
f=[1, \underbrace{0, \ldots, 0}_{m-1 \text { elements }}]
$$

Substituting (12),(13) and (11) into formula (11), we transfer the equation

$y^{4} x+4 y x=\cos 2 x$

into a matrix equation, that is

$$
\begin{aligned}
& c_{m}^{T}\left[I_{m}+4 P_{m}^{4}\right]=d_{m}^{T}-4 f^{T} y^{3} 0 P_{m}^{3} \\
& -4 f^{T} y^{2} 0 P_{m}^{2} \\
& -4 f^{T} y^{\prime} 0 P_{m}-4 f^{T} y 0
\end{aligned}
$$

where $I_{m}$ is identity matrix.

Then, we choose four cases, which involve to the ordinary differential equation (11) to perform the Haar wavelet method.

Case 1 Simple-supported at both ends

Using boundary conditions

$$
\text { y } 0=y^{2} \quad 0=0, y 1=y^{2} 1=0
$$

we can gain

$$
\begin{aligned}
& y^{3} 0=-c_{m}^{T} P_{m} f \\
& y^{\prime} 0=-c_{m}^{T} P_{m}^{3} f+f^{T}\left[c_{m}^{T} P_{m} f\right] P_{m}^{2} f
\end{aligned}
$$

Case 2 Fixed end at one and simple-supported at the other one

Using boundary conditions

$$
\text { y } 0=y^{\prime} 0=0, y 1=y^{2} \quad 1=0
$$

we can gain 


$$
\begin{aligned}
& y^{3} \quad 0=\frac{c_{m}^{T} P_{m} f\left[f^{T} P_{m} f\right]-c_{m}^{T} P_{m}^{3} f}{f^{T} P_{m}^{2} f-f^{T} P_{m} f} \\
& y^{2} \quad 0=-c_{m}^{T} P_{m} f \\
& -\frac{c_{m}^{T} P_{m} f\left[f^{T} P_{m} f\right]-c_{m}^{T} P_{m}^{3} f}{f^{T} P_{m}^{2} f-f^{T} P_{m} f}
\end{aligned}
$$

Case 3 Fixed end at one and free end at the other one

Using boundary conditions

$$
\text { y } 0=y^{\prime} 0=0, y^{2} \quad 1=y^{3} 1=0
$$

we can gain

$$
\begin{aligned}
& y^{3} \quad 0=-c_{m}^{T} f \\
& y^{2} \quad 0=-c_{m}^{T} P_{m} f-c_{m}^{T} f
\end{aligned}
$$

Case 4 Fixed end at both ends

Using boundary conditions

$$
\text { y } 0=y^{\prime} 0=0, y^{2} \quad 1=y^{3} \quad 1=0
$$

we can gain

$$
\begin{gathered}
y^{3} \quad 0=\frac{c_{m}^{T} P_{m}^{2} f\left[f^{T} P_{m} f\right]-c_{m}^{T} P_{m}^{3} f}{f^{T} P_{m}^{2} f-f^{T} P_{m} f^{2}} \\
y^{2} \quad 0=-c_{m}^{T} P_{m}^{2} f \\
-\frac{c_{m}^{T} P_{m}^{2} f\left[f^{T} P_{m} f\right]^{2}-c_{m}^{T} P_{m}^{3} f\left[f^{T} P_{m} f\right]}{f^{T} P_{m}^{2} f-f^{T} P_{m} f^{2}}
\end{gathered}
$$

Substituting $y^{3} 0, y^{2} 0, y^{\prime} 0$ and $y 0$ into formula (19), we can gain $c_{m}^{T}$ via solving the matrix equation,

then we have $y \quad x$ by formula (17) respectively.

The comparison of analytic solution and the Haar solution is shown in Table.2. It is seen that even when $m=32$ and $m=64$ the Haar direct method quite satisfactory.

Table 2 Comparison of the analytic solution and the Haar solution for simple-supported at both ends

\begin{tabular}{cccc}
\hline$x$ & $\begin{array}{c}\text { Exact } \\
\text { solution }\end{array}$ & $\begin{array}{c}\text { Haar } \\
\text { solution } \\
m=32\end{array}$ & $\begin{array}{c}\text { Haar } \\
\text { solution } \\
m=64\end{array}$ \\
\hline 0.0 & 0.0 & 0.0 & 0.0 \\
\hline
\end{tabular}

\begin{tabular}{cccc}
\hline 0.0625 & 0.0013 & 0.0014 & 0.0013 \\
0.1875 & 0.0037 & 0.0038 & 0.0037 \\
0.3125 & 0.0054 & 0.0055 & 0.0054 \\
0.4375 & 0.0061 & 0.0059 & 0.0061 \\
0.5625 & 0.0059 & 0.0060 & 0.0059 \\
0.6875 & 0.0048 & 0.0047 & 0.0048 \\
0.8125 & 0.0031 & 0.0033 & 0.0032 \\
0.9375 & 0.0010 & 0.0011 & 0.0010 \\
1.0 & 0.0 & 0.0 & 0.0 \\
& & & \\
\hline
\end{tabular}

Table 3 Comparison of the analytic solution and the Haar solution for fixed end at one and simple-supported at the other one

\begin{tabular}{cccc}
\hline$x$ & $\begin{array}{c}\text { Exact } \\
\text { solution }\end{array}$ & $\begin{array}{l}\text { Haar } \\
\text { solution }\end{array}$ & $\begin{array}{c}\text { Haar solution } \\
m=64\end{array}$ \\
& & $m=32$ & \\
\hline 0.0 & 0.0 & 0.0 & 0.0 \\
0.0625 & 0.00012 & 0.00014 & 0.00013 \\
0.1875 & 0.00080 & 0.00072 & 0.00078 \\
0.3125 & 0.00160 & 0.00140 & 0.00150 \\
0.4375 & 0.00210 & 0.00200 & 0.00210 \\
0.5625 & 0.00230 & 0.00210 & 0.00230 \\
0.6875 & 0.00200 & 0.00190 & 0.00200 \\
0.8125 & 0.00140 & 0.00130 & 0.00140 \\
0.9375 & 0.00057 & 0.00052 & 0.00056 \\
1.0 & 0.0 & 0.0 & 0.0 \\
\hline
\end{tabular}

Table 4 Comparison of the analytic solution and the Haar solution for fixed end at one and free end at the other end

\begin{tabular}{cccc}
\hline$x$ & $\begin{array}{c}\text { Exact } \\
\text { solution }\end{array}$ & $\begin{array}{c}\text { Haar solution } \\
m=32\end{array}$ & $\begin{array}{c}\text { Haar solution } \\
m=64\end{array}$ \\
\hline & & & \\
0.0 & 0.0 & 0.0 & 0.0 \\
0.0625 & 0.00017 & 0.00012 & 0.00016 \\
0.1875 & 0.00120 & 0.00130 & 0.00120 \\
0.3125 & 0.00260 & 0.00250 & 0.00260 \\
0.4375 & 0.00400 & 0.00380 & 0.00390 \\
0.5625 & 0.00520 & 0.00530 & 0.00510 \\
0.6875 & 0.00620 & 0.00610 & 0.00620 \\
0.8125 & 0.00700 & 0.00690 & 0.00690 \\
0.9375 & 0.00770 & 0.00760 & 0.00770 \\
1.0 & 0.00790 & 0.00780 & 0.00780 \\
\hline
\end{tabular}

Table 5 Comparison of the analytic solution and the Haar solution for fixed end at both ends

\begin{tabular}{cccc}
\hline$x$ & $\begin{array}{c}\text { Exact } \\
\text { solution }\end{array}$ & $\begin{array}{c}\text { Haar solution } \\
m=32\end{array}$ & $\begin{array}{c}\text { Haar solution } \\
m=64\end{array}$ \\
\hline & & & \\
0.0 & 0.0 & 0.0 & 0.0 \\
0.0625 & 0.0001 & 0.0002 & 0.0001 \\
0.1875 & 0.0006 & 0.0005 & 0.0006 \\
0.3125 & 0.0011 & 0.0012 & 0.0011 \\
0.4375 & 0.0014 & 0.0016 & 0.0014 \\
0.5625 & 0.0013 & 0.0012 & 0.0013 \\
\hline
\end{tabular}




\begin{tabular}{cccc}
\hline 0.6875 & 0.0009 & 0.0008 & 0.0009 \\
0.8125 & 0.0004 & 0.0005 & 0.0004 \\
0.9375 & 0.0001 & 0.0002 & 0.0001 \\
1.0 & 0.0 & 0.0 & 0.0 \\
\hline
\end{tabular}

It follows from Tables 2, 3,4 and 5 that already in the cases $m=32$ or $m=64$ we get the results pertaining to our algorithm is closer to analytic solution. The error is negligible.

\section{NUMERICAL TEST}

\section{Illustrative Example:}

A cantilever beam of length $l$ and weighing $\omega l b . /$ unit is subjected to a horizontal compressive force $P$ applied at the free end. Taking the origin at the free end and $y$-axis upwards, we establish the differential equation of the beam

$$
\begin{aligned}
& E I \frac{d^{2} y}{d x^{2}}=-P y-\omega x \cdot \frac{x}{2} \\
& \text { (or) } E I \frac{d^{2} y}{d x^{2}}+P y=-\frac{\omega x^{2}}{2}
\end{aligned}
$$

Solving the above equation, the complete solution is $y=c_{1} \cos n x+c_{2} \sin n x$

$+\frac{\omega}{2 P}\left(\frac{2}{n^{2}}-x^{2}\right)$, where $\frac{P}{E I}=n^{2}$,

The boundary conditions at the fixed end are $x=l, y=\delta$, the maximum deflection $d y / d x=0$.

Using the condition $x=l, y=\delta$, equation (18) gives

$$
\delta=c_{1} \cos n l+c_{2} \sin n l+\frac{\omega}{2 P}\left(\frac{2}{n^{2}}-l^{2}\right) .
$$

Applying the condition $\frac{d y}{d x}=0$, it gives

$0=n-c_{1} \sin n l+c_{2} \cos n l-\frac{\omega l}{P}$

Also imposing the boundary condition for the free end (i.e. $x=0, d^{2} y / d x^{2}=0$ ) on

$$
\frac{d^{2} y}{d x^{2}}=-n^{2} \quad c_{1} \cos n x+c_{2} \sin n x-\frac{\omega}{P},
$$

we get

$$
0=-n^{2} c_{1}-\frac{\omega}{P} \text {, i.e. } c_{1}=-\omega / P n^{2} .
$$

Then $c_{2}=\frac{\omega l}{p n} \sec n l-\frac{\omega}{P n^{2}} \tan n l$

The maximum deflection

$$
\delta=\frac{\omega}{P n^{2}}\left(1-\frac{l^{2} n^{2}}{2}-\sec n l+n l \tan n l\right)
$$

Equation (20) can be written as

$$
y^{2} x+n^{2} y x=\frac{-\omega x^{2}}{2}, \text { where } \frac{P}{E I}=n^{2}
$$

For solving this problem by the Haar wavelet method, we assume that $y^{2} x$ can be expanded in terms of Haar wavelets as formula (2). That is

$$
\begin{aligned}
& y^{2} x=\sum_{n=0}^{m-1} c_{n} h_{n} \quad x=c_{m}^{T} h_{m} x \\
& \text { The function } \frac{-\omega x^{2}}{2} \text { can be expanded into Haar }
\end{aligned}
$$

series over the interval 0,1

$$
\frac{-\omega x^{2}}{2}=\sum_{n=0}^{m-1} d_{n} h_{n} \quad x=d_{m}^{T} h_{m} \quad x
$$

where the vector $d_{m}^{T}$ can be obtained using formula (3). Integrating formula (25) from 0 to $x$ and using formula (5), the variables $y^{\prime} x$ and $y \quad x$ can be expressed as

$y^{\prime} x=\int_{0}^{x} y^{2} t d t+y^{\prime} 0$

$=\left[\begin{array}{l}c_{m}^{T} P_{m}^{3}+f^{T} y^{3} \quad 0 P_{m}^{2} f^{T} y^{2} \quad 0 \quad P_{m} \\ +f^{T} y^{\prime} 0\end{array}\right.$

$h_{m} x$

$$
\begin{aligned}
y \quad x & =\int_{0}^{x} y^{\prime} t d t+y \quad 0 \\
& =\left[\begin{array}{lllll}
c_{m}^{T} P_{m}^{4}+f^{T} y^{3} & 0 & P_{m}^{3} f^{T} y^{2} & 0 \\
P_{m}^{2}+f^{T} y^{\prime} & 0 & P_{m} f^{T} y & 0
\end{array}\right]
\end{aligned}
$$

$h_{m} x$

where the vector $f$ is defined as

$$
f=[1, \underbrace{0, \ldots, 0}_{m-1 \text { elements }}]
$$

Substituting (25), (26) and (28) into formula (24), we transfer the equation

$$
\begin{aligned}
& y^{2} x+n^{2} y x \\
& =\frac{-\omega x^{2}}{2}, \text { where } \frac{P}{E I}=n^{2}
\end{aligned}
$$




$$
\begin{aligned}
& c_{m}^{T}\left[I_{m}+n^{2} P_{m}^{4}\right] \\
& =d_{m}^{T}-n^{2} f^{T} y^{3} \quad 0 \quad P_{m}^{3} \\
& -n^{2} f^{T} y^{2} \quad 0 \quad P_{m}^{2} \\
& -n^{2} f^{T} y^{\prime} \quad 0 \quad P_{m}-n^{2} f^{T} y \quad 0
\end{aligned}
$$

where $I_{m}$ is identity matrix.

From the above formula the wavelet coefficients $c_{(m)}^{T}$ can be successively calculated.

This process is started with

$$
\begin{aligned}
& y x_{l}, t_{s}=\frac{-\omega x^{2}}{2}, \\
& y^{\prime} x_{l}, t_{s}=-\omega x, \\
& y^{\prime \prime} x_{l}, t_{s}=-\omega .
\end{aligned}
$$

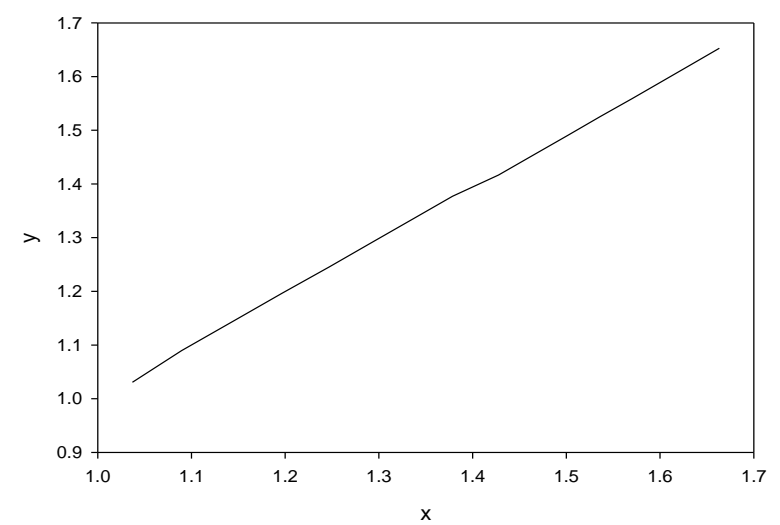

Fig. 2 Comparison between Haar and exact solutions for $m=64$.

Fig.2 shows the comparison between the Haar and exact solutions of equation (18). In this example, $m=64$ is used. If a larger $m$ is chosen, a better estimation is expected.

\section{CONCLUSION}

The main goal of this paper was to demonstrate that the Haar wavelet method is a powerful tool for solving finite length beam differential equation. The algorithm and procedure have been applied to use Haar wavelet method in solving ODEs. The result is comparable to the exact solution. The method with far less degrees of freedom and with smaller CPU time provides better solutions than classical ones.

It is worth mentioning that Haar solution provides excellent results even for small values of $m$ ie., $m=64$. For larger values of $m$, we can obtain the results closer to the real values. The main advantages of the presented method are its simplicity and small computation costs: it is due to the sparcity of the transform matrices and to the small number of significant wavelet coefficients. The method is also very convenient for solving the boundary value problems, since the boundary conditions are taken care of automatically. In our opinion the method is wholly competitive in comparison with the classical methods.

\section{REFERENCES}

[1] L.AlvarezDíaz, M.T.Martín, V.Vampa, Daubechies wavelet beam and plate finite elements, Finite Elements in Analysis and Design 45 (2009) 200-209.

[2] M. A. Biot, J. appl. Mech. A.S.M.E. 4, A-1 (1937).

[3] C. Cattani, Haar wavelet spline, J.Interdisciplinary Math.4 (2001), 35-47.

[4] C.F.Chen, C.H.Hsiao, Haar wavelet method for solving lumped and distributed-parameter systems, IEEE Proc.Pt.D 144(1) (1997) 87-94.

[5] R. Dai , J. E. Cochran, Wavelet Collocation Method for Optimal Control Problems, J Optim. Theory Appl. doi 10.1007/s10957-009-9565-9.

[6] A. Haar, Zur theorie der orthogonalen Funktionsysteme.Math. Annal 69:(1910) 331-371.

[7] G. Hariharan, K.Kannan, Kal Renganathan Sharma., Haar wavelet in estimating depth profile of soil temperature, Appl. Math. Comput. 210, (2009) 119-125.

[8] M. Hetdnyi, Beams on Elastic Foundation: Theory with Applications in the Fields of Civil and Mechanical Engineering, The University of Michigan Press, Ann Arbor, MI, 1946 ( printing: 1993).

[9] C.H. Hsiao, W.J.Wang, Haar wavelet approach to nonlinear stiff systems, Math.Comput.Simulat, 57, (2001) pp.347353.

[10] C.H.Hsiao, Haar wavelet approach to linear stiff systems, Mathematics and Computers in simultion ,Vol 64, 2004, pp.561-567.

[11] U. Lepik, Dynamic response of elastic-plastic beams with axial constraints. Int. J. Impact Engg 15, 3-16 (1994).

[12] U. Lepik, Impulsively loaded fully fixed-ended elasticplastic beams by Galerkin's method. Int. J. Impact Engng 15, 17-23 (1994).

[13] U. Lepik, Vibrations of elastic-plastic fully clamped beams and flat arches under impulsive loading. Int. J.on-Linear Mech. 29, 613-623 (1994).

[14] U.Lepik, Numerical solution of differential equations using Haar wavelets, Math. Computers in Simulation 68 (2005) 127-143.

[15] U.Lepik, Numerical solution of evolution equations by the Haar wavelet method, Appl. Math. Comput. 185 (2007) 695-704.

[16] U. Lepik, Application of the Haar wavelet transform to solving integral and differential Equations, Proc.EstonianAcad. Sci.Phys.Math., 56, 1, (2007)28-46.

[17] M.N. Pavlovid, S.Tsikkos, Beams on quasi-Winkler foundations, Eng. Struct, Vol. 4, (1982).

[18] E. Winkler, Die Lehre yon der Elastizitat und FestigIceit, $p$. 182. Prague (1867)

[19] G.P. Xia, Z. Zhang, A Numerical Method for Critical Buckling Load for a Beam Supported Elastic Foundation, EJGE, 1-11(2009). 